\title{
Microscopic Origins of the Anomalous Melting Behavior of Sodium under High Pressure
}

Eshet, Hagai ; Khaliullin, Rustam Z ; Kuehne, Thomas D ; Behler, Joerg ; Parrinello, Michele

\begin{abstract}
X-ray diffraction experiments have shown that sodium exhibits a dramatic pressure-induced drop in melting temperature, which extends from $1000 \mathrm{~K}$ at similar to $30 \mathrm{GPa}$ to as low as room temperature at similar to $120 \mathrm{GPa}$. Despite significant theoretical effort to understand the anomalous melting, its origins are still debated. In this work, we reconstruct the sodium phase diagram by using an ab initio quality neural-network potential. Furthermore, we demonstrate that the reentrant behavior results from the screening of interionic interactions by conduction electrons, which at high pressure induces a softening in the short-range repulsion.
\end{abstract}

DOI: https://doi.org/10.1103/PhysRevLett.108.115701

Posted at the Zurich Open Repository and Archive, University of Zurich ZORA URL: https://doi.org/10.5167/uzh-65269

Journal Article

Originally published at:

Eshet, Hagai; Khaliullin, Rustam Z; Kuehne, Thomas D; Behler, Joerg; Parrinello, Michele (2012). Microscopic Origins of the Anomalous Melting Behavior of Sodium under High Pressure. Physical Review Letters, 108(11):115701.

DOI: https://doi.org/10.1103/PhysRevLett.108.115701 


\title{
Microscopic origins of the anomalous melting behaviour of high-pressure sodium
}

\author{
Hagai Eshet, ${ }^{1, *}$ Rustam Z. Khaliullin, ${ }^{1,2, \dagger}$ Thomas D. Kühne, ${ }^{3,4}$ Jörg Behler, ${ }^{5}$ and Michele Parrinello ${ }^{1,6}$ \\ ${ }^{1}$ Department of Chemistry and Applied Biosciences, ETH Zürich, \\ USI Campus, via G. Buffi 13, 6900 Lugano, Switzerland \\ ${ }^{2}$ Physical Chemistry Institute, University of Zürich, \\ Winterthurerstrasse 190, 8057 Zürich, Switzerland \\ ${ }^{3}$ Institute of Physical Chemistry, Johannes Gutenberg University Mainz, D-55128 Mainz, Germany \\ ${ }^{4}$ Center for Computational Sciences, Johannes Gutenberg University Mainz, D-55128 Mainz, Germany \\ ${ }^{5}$ Lehrstuhl für Theoretische Chemie, Ruhr-Universität Bochum, D-44780 Bochum, Germany \\ ${ }^{6}$ Facoltà di Informatica, Istituto di Scienze Computazionali, \\ Università della Svizzera Italiana, via G. Buffi 13, 6900 Lugano, Switzerland
}

(Dated: February 1, 2012)

\begin{abstract}
X-ray diffraction experiments have shown that sodium exhibits a dramatic pressure-induced drop in melting temperature, which extends from $1000 \mathrm{~K}$ at $\sim 30 \mathrm{GPa}$ to as low as room temperature at $\sim 120 \mathrm{GPa}$. Despite significant theoretical effort to understand the anomalous melting its origins are still debated. In this work, we reconstructed the sodium phase diagram using an ab-initioquality neural-network potential. Further, we demonstrated that the reentrant behaviour results from the screening of interionic interactions by conduction electrons, which at high pressure induces a softening in the short-range repulsion.
\end{abstract}

Sodium, a prototype simple metal at ambient conditions, exhibits unexpected complexity under high pressure [1-6]. At ambient conditions, sodium crystallizes in the body-centered cubic (bcc) structure, which transforms to the face-centered cubic (fcc) phase upon compression to $65 \mathrm{GPa}$ [7]. At $105 \mathrm{GPa}$, the fcc phase undergoes a transformation to the cI16 phase - a distorted variant of the bcc structure $[2,8]$. Further compression results in the formation of a large variety of complex phases $[3-6]$, the existence of which is quite intriguing. However, the focus of this work is one of the most striking features of the sodium phase diagram - the anomalous melting curve. Diffraction measurements have revealed a maximum in melting temperature around $1000 \mathrm{~K}$ at $\sim 30 \mathrm{GPa}$ followed by a pressure-induced drop, which extends to nearly room temperature at $\sim 120 \mathrm{GPa}[1]$ and over the stability regions of three solid phases (Fig. 1a). This anomaly implies that in this range the liquid is denser than the solid.

Although previous ab initio studies [9-14] have reproduced the reentrant melting behaviour of sodium the physical origin of this anomaly still remains unclear. The hypothesis of structural and electronic transitions in liquid sodium proposed in one of the studies [10] has not been confirmed by other authors [9].

It is important to point out that due to the high computational cost of $a b$ initio MD all simulations so far reported [9-12] have used small simulation cells and estimated melting temperatures $\left(T_{m}\right)$ by heating the solid phase until it melts. In such simulations, the absence of nucleation centers and fast heating schedules delay melting relative to the thermodynamic coexistence point and, therefore, only an upper bound on $T_{m}$ can be obtained. A proper reconstruction of the melting curve requires comparing the free energies of the liquid and solid phases

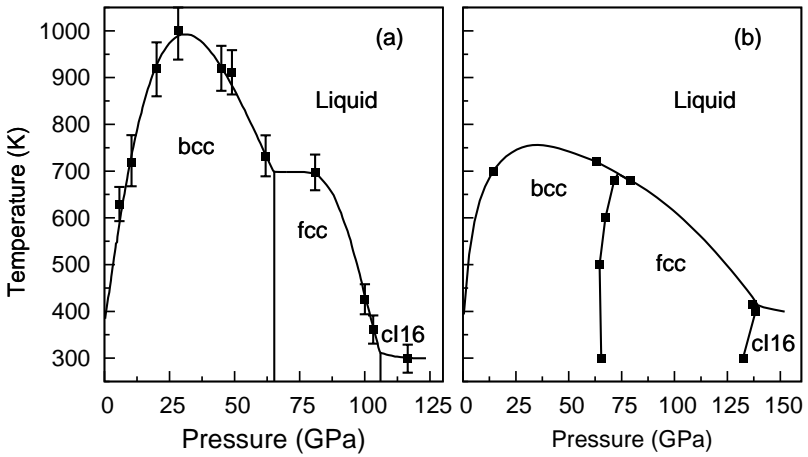

FIG. 1. Sodium phase diagram. a. The squares represent experimentally measured melting points. The lines show tentative phase boundaries [1]. b. Theoretical results based on the NN potential. The squares represent coexistence points computed with thermodynamic integration. The lines show coexistence curves traced by integrating the Clausius-Clapeyron equation (see Supplementary Information).

and involves long ( $\sim 10 \mathrm{~ns})$ MD simulations on large systems $\left(\sim 10^{3}\right.$ atoms $)$. Performing such simulations with direct ab initio methods is at present computationally too expensive. Although several authors [13, 14] have demonstrated that simplified models can also reproduce the anomalous melting behaviour the relation of these models to the actual physical interactions in sodium has remained unclear.

In a previous work [15], we have shown that a neuralnetwork $(\mathrm{NN})$ potential $[16,17]$ for sodium based on wellconverged Perdew-Burke-Ernzerhof [18] density functional calculations retains the accuracy of ab initio simulations at a highly reduced computational cost. The ability of the NN potential to reproduce numerous $a b$ 
initio and experimental properties of the solid and the liquid phases in the pressure range up to $140 \mathrm{GPa}$ has been demonstrated in Ref. 15. Additionally, Supplementary Fig. 1 shows the high accuracy of the NN potential in reproducing the $a b$ initio energies for liquid sodium structures along $\mathrm{MD}$ trajectories in the relevant $\mathrm{P}-\mathrm{T}$ range. Furthermore, Supplementary Fig. 2 demonstrates that the "heat-until-it-melts" melting curve calculated with the NN potential is in good agreement with that obtained from ab initio simulations [12].

Here we utilized the NN potential to construct the sodium phase diagram. The phase diagram shown in Fig. 1 was calculated by locating points of equal chemical potential for each pair of phases in the $\mathrm{P}-\mathrm{T}$ plane. This was done in three steps (see Ref. 19 and Supplementary Information). First, we calculated the Helmholtz free energy of each pair of phases by thermodynamic integration using the Einstein crystal and the Lennard-Jones potential as the reference systems [20]. In the next step, the chemical potentials were evaluated by integrating the free energy as a function of the density. Finally, the coexistence lines were traced by integrating the ClausiusClapeyron equation using the predictor-corrector scheme of Kofke [21]. The total simulation time required to model the phase diagram amounts to 50 ns clearly demonstrating the computational advantage of the NN approach in comparison with the direct ab initio simulation.

The NN potential captures the experimentally observed sequence of the solid-state phase transitions $\mathrm{bcc} \rightarrow \mathrm{fcc} \rightarrow \mathrm{cI} 16$ and the regions of stability of each phase. The shape of the melting curve with the maximum around $30 \mathrm{GPa}$ is also correctly reproduced. The comparison of NN and DFT transition pressures at zero temperature (Fig. 6 in Ref. 15) as well as the "heatuntil-it-melts" melting curves obtained from NN and $a b$ initio simulations (Supplementary Fig. 2) show that the quantitative errors in the coexistence lines can be attributed to inaccuracies of the density functional and not to the errors of the NN fitting. Supplementary Fig. 2 also demonstrates that $T_{m}$ obtained with a non-equilibrium approach is overestimated compared to the proper thermodynamic calculation. To the best of our knowledge this is the first phase diagram calculated from a highquality ab initio method.

To verify the existence of structural and electronic transitions in the liquid phase, which has been suggested before to explain the anomalous melting [10], we evaluated the order parameter introduced in Ref. 10 to monitor structural changes in the liquid sodium (see Supplementary Information). Our calculations do not reveal any significant variations in the structure of liquid in the 0-140 GPa range along the calculated melting curve (Supplementary Fig. 3a). In addition, the electronic density of states calculated with DFT for liquid structures collected from NN-driven MD simulations does not ex- hibit the opening of a pseudogap observed before [10] (Supplementary Fig. 3b). Discrepancies between our results and Ref. 10 can be attributed to the explicit inclusion of the $2 s$ and $2 p$ electrons in the present calculations. It has been shown that these states play an important role in determining properties of liquid at high pressure [12].

While the NN simulations reproduce adequately the sodium melting curve the physical origins of its reentrant behaviour are difficult to extract. To this effect, we constructed a density-dependent pair potential based on the jellium model for the conducting electrons. According to this model the ionic structure of a metal can be replaced, to a first approximation, by a uniform positivelycharged background while conduction electrons can be treated as a uniform electron gas. The granularity of sodium is then introduced at the level of two-body interactions by immersing two $\mathrm{Na}$ atoms in such a jellium and calculating the energy as a function of the interatomic distance (see Supplementary Information). The effect of the pressure is taken into account by varying the density of the electron gas $\rho_{e}$ according to the NN equation of state. By doing so, we retraced a time-honoured idea used to construct effective potentials describing screening effects in metals by linear response theory [22]. Here, we used a non-linear approach since it is expected that non-linear screening effects become important as the ions are brought closer to each other by the applied pressure.

We found that the two-body effective potential obtained from the jellium model is well reproduced by a sum of the repulsive Yukawa potential and the oscillatory Friedel term (Supplementary Fig. 4) [23, 24]:

$$
\phi(r)=\frac{A \exp \left(-k_{0} r\right)}{r}+\frac{B \cos \left[2 k_{F}\left(r-r_{0}\right)\right]}{r^{m}},
$$

where $A, B, k_{0}, r_{0}$ and $m$ are density dependent fitting parameters and $k_{F}$ is the true (i.e. not fitted) Fermi wavenumber (see Supplementary Table 1). This analytical form of the potential is inspired by a linear screening theory [22] and reflects the nature of physical interactions in metallic sodium, in which the direct $\mathrm{Na}-\mathrm{Na}$ repulsion is screened by the free-electron gas. In particular, the oscillatory term has its origin in the sharpness of the Fermi surface.

MD simulations based on the effective pair potential reproduce semi-quantitatively the maximum in the melting curve (Fig. 2). The potential is also remarkably accurate in describing the radial distribution functions (RDFs) of the liquid for pressures up to $100 \mathrm{GPa}$ (Supplementary Fig. 5). We thus believe that this effective density dependent two-body potential is able to capture the physics of the problem and can be used to shed light on the origin of the anomalous melting behaviour. It is worth mentioning that the validity of this potential is limited to the region of pressures below about $120 \mathrm{GPa}$ because beyond this region electrons are promoted to the $d$-states 


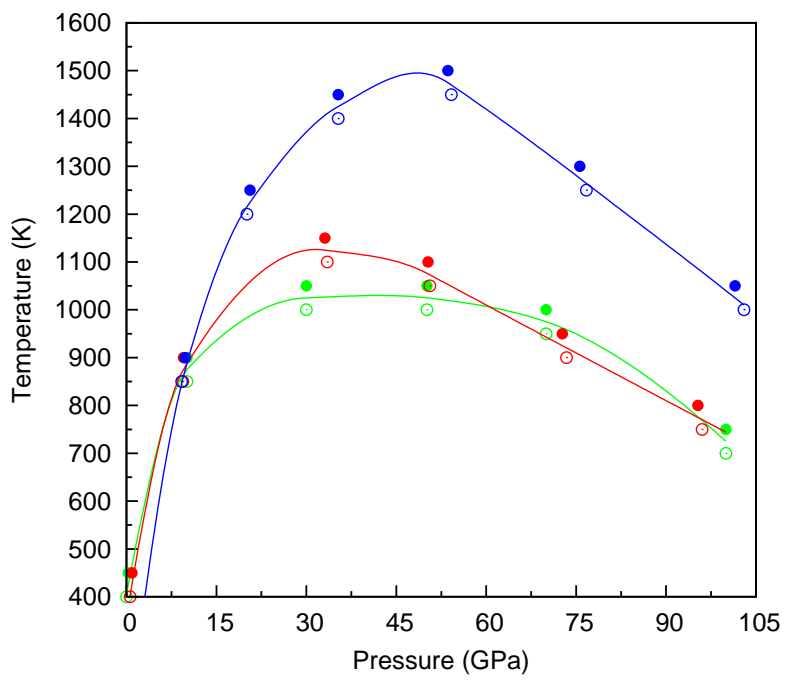

FIG. 2. The "heat-until-it-melts" curves obtained with the NN potential (green), effective pair potential based on the jellium model (red) and repulsive wall of the effective pair potential (blue). The points below $90 \mathrm{GPa}$ are obtained by melting the bcc phase, the points above $90 \mathrm{GPa}$ by melting the fcc phase. To obtain the curves temperature was increased in $50 \mathrm{~K}$ increments with open and solid circles showing the solid and liquid states, respectively.

and the simple free-electron two-body model is no longer appropriate.

We examined the effect of two different parts of the effective potential on the melting curve. First, we considered the Yukawa part of the potential and, second, the repulsive wall of $\phi(r)$. The latter was constructed by truncating the total potential at its first minimum and shifting it so that both the energy and force are zero at the truncation point [25]. We found that the Yukawa potential cannot reproduce the reentrant melting behaviour. On the contrary, the melting curve obtained with the repulsive wall exhibits a maximum similar to that obtained with the full potential (Fig. 2). This observation suggests that it is the short range repulsive part of the potential that provides the origin of the anomalous melting (see Supplementary Information for a detailed pressure decomposition analysis that confirms this conclusion).

The log-log plot of the interatomic force in the region of the repulsive potential wall shown in Fig. 3 supports this point of view. It is seen that there is a large change of the slope as the potential changes from $1 / r^{12}$ to a much softer $1 / r^{3}$ behaviour (i.e. the slope changes from $1 / r^{13}$ to $1 / r^{4}$ in Fig. 3 ). The lower panel of the same figure shows the RDFs obtained at different pressures along the melting line. It demonstrates that at low pressure only the steep part of the repulsive wall is sampled, whereas at higher pressure the softer short-range part of the potential starts influencing the atomic interactions. Since softening the potential lowers the melting tempera-

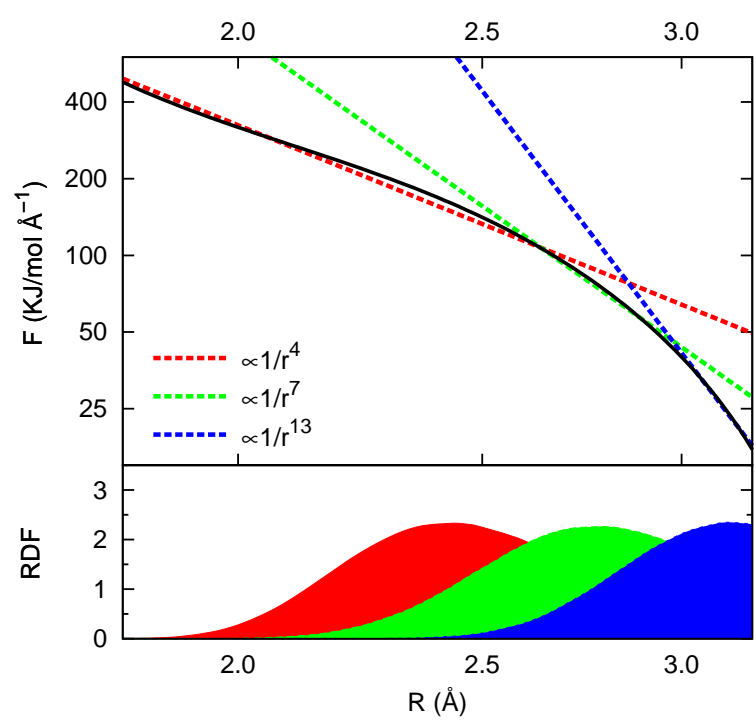

FIG. 3. Upper panel: interatomic forces in the region of the repulsive wall of the effective pair potential (black solid line). Lower panel: RDF of liquid sodium along the melting curve at $\sim 10 \mathrm{GPa}$ (blue), $\sim 30 \mathrm{GPa}$ (green) and $\sim 90 \mathrm{GPa}$ (red).

ture $[26,27]$ we argue that the softening of the repulsive interactions in sodium at high pressure is the origin of the observed anomaly.

Our results fit well into the existing theories of metals that predict an increase of screening with density [28, 29]. They are also in agreement with theoretical predictions that certain softening in simple model potentials can result in anomalous melting [30]. Our work demonstrates, for the first time, that the screening-induced softening can lead to a dramatic decrease in the melting temperature of a real material.

In order to understand the electronic origin of the softening effect we examined the behaviour of the electron localization function (ELF), which is widely used to analyse the nature of chemical bonds (see Methods) [31, 32]. Fig. 4a shows the behaviour of the derivative of the ELF with respect to the interatomic distance, which can be taken as a measure of the softness of the bond. The derivative closely follows the behaviour of the melting curve dramatically decreasing at high pressure. It is instructive to compare the ELFs at low and high pressures. Fig. 4b shows that electrons localized in the $\mathrm{Na}-\mathrm{Na}$ bond move to the outer regions as pressure increases. The tendency of electrons to move from the bonding regions to the interstitial regions at high pressure has already been noted [33, 34].

In conclusion, the insight into the electronic and structural properties of sodium obtained in this work offers a new consistent explanation of the anomalous melting behaviour of $\mathrm{Na}$. We demonstrated that the observed dramatic drop in the melting temperature can be attributed 


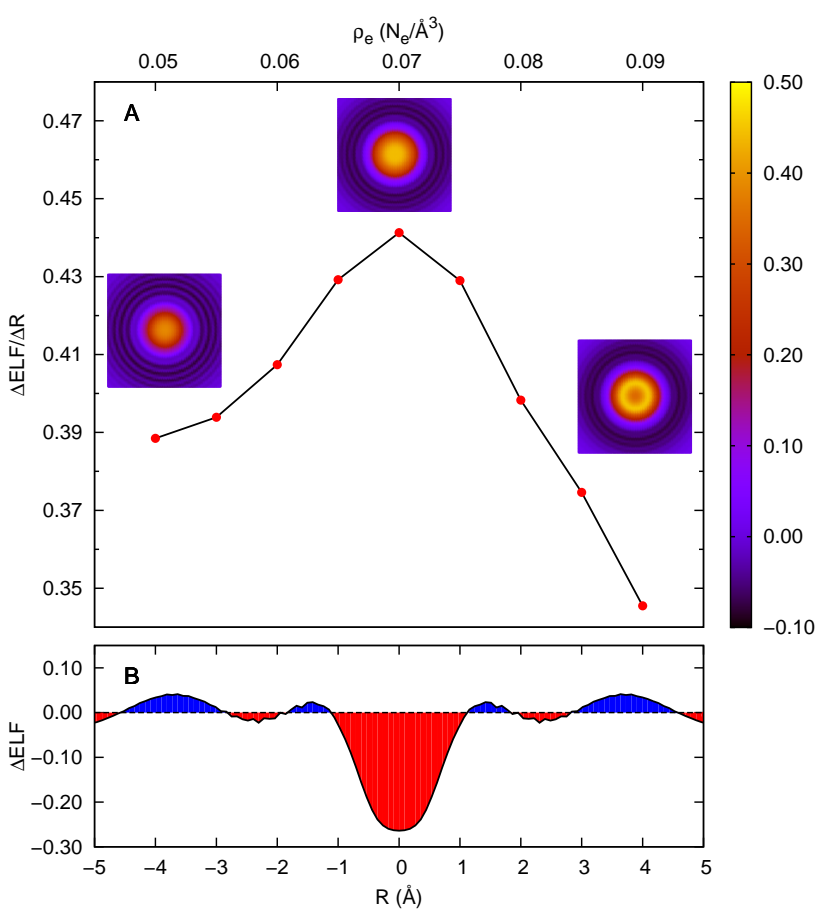

FIG. 4. a. Pressure dependence of the derivative of the ELF at the center of the $\mathrm{Na}-\mathrm{Na}$ bond wrt interatomic distance taken at the distance of the nearest neighbour in the bcc lattice. For comparison, the maximum of $T_{m}$ is around $\rho_{e} \approx 0.0615 \AA^{-3}$. The insets show $2 \mathrm{D}$ plots of the same derivative in the plane perpendicular to the $\mathrm{Na}-\mathrm{Na}$ bond for $\rho_{e}$ $0.05 \AA^{-3}(\sim 20 \mathrm{GPa}), 0.07 \AA^{-3}(\sim 45 \mathrm{GPa})$ and $0.09 \AA^{-3}$ $(\sim 90 \mathrm{GPa})$. b. Difference between the high-pressure and low-pressure values of ELF in the direction perpendicular to the $\mathrm{Na}-\mathrm{Na}$ bond, $\triangle \mathrm{ELF} \equiv \mathrm{ELF}(90 \mathrm{GPa})-\mathrm{ELF}(25 \mathrm{GPa})$.

to the density dependence of the conduction electrons screening, which induces a softening of the interatomic potential at short range. Our preliminary results indicate that similar softening effects are responsible for the reentrant melting in other alkali metals such as lithium and potassium. These findings have immediate implications for explaining behaviour of other metals and alloys.

Acknowledgments. This work was supported by the European Research Council (ERC-2009-AdG-247075). J.B. is grateful for financial support from the FCI and the DFG. T.D.K. would like to thank the Graduate School of Excellence MAINZ and the IDEE project of the Carl Zeiss Foundation for financial support. Our thanks are also due to the Swiss National Supercomputing Centre (CSCS) and High Performance Computing Group of ETH Zürich for computer time.

\footnotetext{
* hagai.eshet@gmail.com

† rustam@khaliullin.com
}

[1] E. Gregoryanz, O. Degtyareva, M. Somayazulu, R. J. Hemley, and H. K. Mao, Phys. Rev. Lett. 94, 185502 (2005).

[2] M. I. Mcmahon, E. Gregoryanz, L. F. Lundegaard, I. Loa, C. Guillaume, R. J. Nelmes, A. K. Kleppe, M. Amboage, H. Wilhelm, and A. P. Jephcoat, Proc. Natl. Acad. Sci. U. S. A. 104, 17297 (2007).

[3] E. Gregoryanz, L. F. Lundegaard, M. I. Mcmahon, C. Guillaume, R. J. Nelmes, and M. Mezouar, Science 320, 1054 (2008).

[4] Y. M. Ma, M. Eremets, A. R. Oganov, Y. Xie, I. Trojan, S. Medvedev, A. O. Lyakhov, M. Valle, and V. Prakapenka, Nature 458, 182 (2009).

[5] A. Lazicki, A. F. Goncharov, V. V. Struzhkin, R. E. Cohen, Z. Liu, E. Gregoryanz, C. Guillaume, H. K. Mao, and R. J. Hemley, Proc. Natl. Acad. Sci. U. S. A. 106, 6525 (2009).

[6] L. F. Lundegaard, E. Gregoryanz, M. I. Mcmahon, C. Guillaume, I. Loa, and R. J. Nelmes, Phys. Rev. B 79, 064105 (2009).

[7] M. Hanfland, I. Loa, and K. Syassen, Phys. Rev. B 65, 184109 (2002).

[8] K. Syassen, in High pressure phenomena, proceedings of the international school of physics, edited by R. J. Hemley, G. L. Chiarotti, M. Bernasconi, and L. Ulivi (IOS Press, Amsterdam, -2002-) pp. 266-268.

[9] E. R. Hernandez and J. Iniguez, Phys. Rev. Lett. 98, 055501 (2007).

[10] J. Y. Raty, E. Schwegler, and S. A. Bonev, Nature 449, 448 (2007).

[11] L. Koci, R. Ahuja, L. Vitos, and U. Pinsook, Phys. Rev. B 77, 132101 (2008).

[12] A. Yamane, F. Shimojo, and K. Hoshino, J. Phys. Soc. Jpn. 77, 064603 (2008).

[13] M. Martinez-Canales and A. Bergara, J. Phys. Chem. Solids 69, 2151 (2008).

[14] S. V. Lepeshkin, M. V. Magnitskaya, and E. G. Maksimov, Jetp Letters 89, 586 (2009).

[15] H. Eshet, R. Z. Khaliullin, T. D. Kühne, J. Behler, and M. Parrinello, Phys. Rev. B 81, 184107 (2010).

[16] J. Behler and M. Parrinello, Phys. Rev. Lett. 98, 146401 (2007).

[17] J. Behler, Phys. Chem. Chem. Phys. 13, 17930 (2011).

[18] J. P. Perdew, K. Burke, and M. Ernzerhof, Phys. Rev. Lett. 77, 3865 (1996).

[19] R. Z. Khaliullin, H. Eshet, T. D. Kühne, J. Behler, and M. Parrinello, Phys. Rev. B 81, 100103 (2010).

[20] D. Frenkel and B. Smit, Understanding molecular simulation: From algorithms to applications (Academic Press, Orlando, 2001).

[21] D. A. Kofke and P. T. Cummings, Mol. Phys. 92, 973 (1997).

[22] N. W. Ashcroft and N. D. Mermin, Solid state physics (Saunders College, Philadelphia, 1976).

[23] H. R. Leribaux and M. H. Boon, Phys. Rev. B 11, 2412 (1975).

[24] A. Paskin and A. Rahman, Phys. Rev. Lett. 16, 300 (1966).

[25] J. D. Weeks, D. Chandler, and H. C. Andersen, J. Chem. Phys. 54, 5237 (1971).

[26] W. G. Hoover, S. G. Gray, and K. W. Johnson, J. Chem. Phys. 55, 1128 (1971).

[27] D. A. Young, Phase diagrams of elements (University of California Press, Berkeley, 1991). 
[28] J. Hafner and V. Heine, Journal of Physics F-Metal Physics 13, 2479 (1983).

[29] J. Hafner, J. Phys.: Condens. Matter 2, 1271 (1990).

[30] S. Prestipino, F. Saija, and G. Malescio, J. Chem. Phys. 133, 144504 (2010).

[31] A. D. Becke and K. E. Edgecombe, J. Chem. Phys. 92,
5397 (1990).

[32] B. Silvi and A. Savin, Nature 371, 683 (1994).

[33] B. Rousseau and N. W. Ashcroft, Phys. Rev. Lett. 101, 046407 (2008).

[34] J. Yang, J. S. Tse, and T. Iitaka, J. Phys.: Condens. Matter 22, 095503 (2010). 